\title{
Parameterized Mesoscale Forcing Mechanisms for Initiating Numerically Simulated Isolated Multicellular Convection
}

\author{
AdRIAN M. LOFTUS* \\ School of Meteorology, University of Oklahoma, Norman, Oklahoma \\ DANIEL B. WEBER \\ Center for Analysis and Prediction of Storms, University of Oklahoma, Norman, Oklahoma \\ Charles A. Doswell III \\ Cooperative Institute for Mesoscale Meteorological Studies, University of Oklahoma, Norman, Oklahoma
}

(Manuscript received 9 January 2007, in final form 8 November 2007)

\begin{abstract}
Two methods designed to parameterize mesoscale ascent in a three-dimensional numerical cloud model via near-surface momentum and heat fluxes are presented and compared to the commonly used technique of an initial perturbation placed within the model initial condition. The flux techniques use a continuously reinforced thermal or convergent low-level wind field to produce upward vertical motion on the order of 10 $\mathrm{cm} \mathrm{s}^{-1}$, by which deep, moist convection can be initiated. The sensitivity of the convective response to the type, strength, and size of the forcing is evaluated using numerical simulations of a conditionally unstable environment with weak unidirectional shear. Precipitation-free cloud processes are used to further simplify the model response to the forcing. The three methods tested produce an initial convective response, but only the momentum and heat flux methods are able to produce sustained deep convection that approximately resembles isolated multicellular convection. Cell regeneration periods, defined as the elapsed time between subsequent vertical velocity maxima passing through a constant level in the updraft region above the source, vary from 8 to $25 \mathrm{~min}$, depending on the forcing type, magnitude, and geometry.
\end{abstract}

\section{Introduction}

The use of three-dimensional numerical models to simulate deep, moist convection began in the 1970s (for a review see Wilhelmson and Wicker 2002, hereafter WW02). Traditionally, such models in an idealized framework have been initialized with horizontally homogeneous initial conditions, so that some method for initiating the convective cloud is required. In the early studies (e.g., Schlesinger 1975; Klemp and Wilhelmson 1978), an initiating "bubble" (IB) was introduced

\footnotetext{
* Current affiliation: Department of Atmospheric Science, Colorado State University, Fort Collins, Colorado.

Corresponding author address: Dr. Daniel B. Weber, 2311 Blue Court, Norman, OK 73026.

E-mail: daniel.weber@tinker.af.mil
}

within the otherwise horizontally homogeneous atmosphere at the initial time, and the solution was allowed to evolve according to the model's governing equations.

The usual IB involves a thermal and/or moisture perturbation with some prescribed geometry that was never intended to be comparable to a "thermal" developing in a convective boundary layer (see Tripoli and Cotton 1980; McNider and Kopp 1990). Instead, the IB typically is several kilometers in the horizontal scale and hundreds of meters in the vertical scale, such that the IB is often much wider than typical boundary layer thermals observed in nature, which are usually less than $1 \mathrm{~km}$ in horizontal extent (Crum et al. 1987; Young 1988). After the IB is "imposed" at the initial time ( $t=$ 0 ) in the simulation, new deep convection might (or might not) develop along the outflow produced by the first deep convective cloud, and it is assumed that any subsequent deep convective cloud developments were not overly dependent on the nature of the artificial ini- 
tiation mechanism. In some studies (Stensrud et al. 1999), the IB was replaced by an artificially introduced cold pool.

The IB mechanism was never intended to replicate the real atmosphere's processes for initiating convective storms. Presumably, to do so in a physically realistic way, the model would have to include processes on scales well above that of a deep convective cloud. To include those processes directly, as well as the convective cloud itself, requires either (a) computational grids with grid spacings of $1 \mathrm{~km}$ or less over domains encompassing several thousand kilometers, which demands computational resources beyond even present-day capabilities, or (b) grid nesting procedures that can introduce considerable complications to the process. For many purposes, the simple, idealized cloud models have been very useful at increasing our understanding of many aspects of deep convective clouds, as described by WW02.

As presented in WW02, a great deal of the research using these idealized models has been focused on supercells and lines of convective storms, as well as their attendant phenomena. However, relatively little research using idealized cloud models has been done concerning isolated multicellular convective storms, at least partly because it has proven to be difficult to produce such storms when the model begins with an IB in a horizontally homogeneous environment. Here, we define isolated multicellular convection as discrete, periodic, or aperiodic recurring convective cells moving through an updraft region that are not supercells and are not associated with linear or clusters of convective cells. Under some circumstances, such storms can develop along outflows produced by the first deep convective storm originating from the IB, but using this method, it has proven difficult to produce such storms at will. In the real atmosphere, convection begins typically in regions of sustained mesoscale ascent (Doswell 1987), sometimes in a location that is far from being horizontally homogeneous (such as a front or dryline), and sometimes in a mesoscale region of forced ascent (by orography or by localized heating). Thus, we surmised that the IB method of initiating a deep convective cloud in an idealized cloud model might be responsible for the inability to produce isolated nonsupercellular storms easily.

This study represents a first stage in the development of an understanding of the external factors that control the frequency of new updraft "pulses" in a deep convective cloud during the first few hours of convection. Even supercell storms (real or simulated) are not perfectly steady, but typically involve a series of updraft pulsations that manifest themselves by, among other features, episodic changes in the storm-top height. We believe that these pulses are critical to the simulation of multicell storms, and to explore this topic we develop methods herein to produce isolated multicell storms. What appeared to be a promising avenue to follow, therefore, was to replace the IB with a mechanism for producing sustained ascent. For simplicity, it was assumed that the time scale of such ascent would be much longer than that of a deep convective cloud, which has a time scale associated with how long it takes an air parcel to rise through the depth of the cloud (of order $20 \mathrm{~min}$ ). Thus, at least to begin the study, we assumed that the forcing for ascent would be constant in time; this is not necessary, but it does represent a useful simplification. Further, we assumed that the resulting ascent would be of the order of $10 \mathrm{~cm} \mathrm{~s}^{-1}$ (or larger), which corresponds to a value associated with a mesoscale process (Doswell 1987). Finally, to avoid the complication of new convective clouds developing on cold pool boundaries, precipitation was turned off in the simulations. This rather drastic simplification is certainly not realistic, but it allows us to focus on the processes by which the forced ascent produces multiple updraft pulses.

The following two methods for producing this ascent are proposed: one uses a heat source similar to the IB method that, instead of being imposed at $t=0$, is maintained constant in time; the other imposes a constant low-level convergence field that creates a sustained ascent region via mass continuity. These two methods are intended to mimic a sustained heat source (like an island heated by the sun), or a dynamic mesoscale process that generates a localized peak in ascent at low levels (like a dryline). In the first case, a localized continuing source of low-level thermal buoyancy (see Doswell and Markowski 2004) is produced, whereas in the second, the ascent modifies the environment, possibly lifting low-level air to its level of free convection (LFC). Although our initiating mechanisms are not much more physical than the IB method, they do represent two distinctly different processes for producing sustained ascent that bear some resemblance to real processes.

The results presented herein support our hypothesis that forced sustained ascent can generate isolated multicellular convective clouds that resemble such storms in the real atmosphere. Furthermore, we demonstrate that parameterized mesoscale forcing can produce reasonably realistic updrafts, and that our results are sensitive to the details of the forcing. As a first step in a larger study of the effects of environmental parameters on the frequency of updraft pulsations, this article is not aimed at a thorough treatment of the interaction of 
sustained forcing with deep convection; instead, this interaction will be the subject of research yet to be completed. Our methodology also does not produce sustained ascent (simulating a mesoscale process), which is proposed as the optimum way to initiate deep convective clouds in an idealized cloud model.

Section 2 describes the constant forcing methods and the implementation within the numerical framework. Section 3 provides the experimental setup and results for simulated convection for the $0-2$-h time scale. Section 4 provides conclusions and proposes future modifications and applications of the forcing mechanisms.

\section{Specification of convection initiation methods within a numerical cloud model}

Most idealized numerical cloud model studies do not incorporate synoptic-scale or mesoscale forcing as convection initiation mechanisms, but rather rely on IBs or cold pools to initiate deep convection in the simulation. We present three mechanisms used in this study for initiating convection in a numerical cloud model.

The three-dimensional nonhydrostatic numerical cloud model ARPI (Weber 1997), which is similar to ARPS ("AR") but solves the nondimensional pressure ("PI") or Exner function, was developed at the Center for Analysis and Prediction of Storms (CAPS), and used in the present study to perform the simulations of deep, moist convection in the presence of mesoscale forcing. This fully compressible model is based largely on the Advanced Regional Prediction System (ARPS) model developed by CAPS at the University of Oklahoma (see Xue et al. 2000), and was originally designed to test the various types of boundary conditions available in ARPS. It has since been optimized for speed of execution and for use as a numerical cloud model for sensitivity studies. The initialization of the horizontally homogeneous base state and perturbation variables follows that of the ARPS model. The model uses a vertically implicit solution technique, to advance the vertical velocity and nondimensional pressure, and a linearized upper-radiation condition between vertical velocity and nondimensional pressure. In addition, the nondimensional Exner function is solved in this model instead of pressure, unlike ARPS. The model solution process is spilt into two time steps-a large time step, which integrates the slow modes (gravity waves, advection, mixing processes) for all variables, and a small time step for pressure gradient and compressibility effects. The leapfrog time scheme computational modes are damped using an Asselin (1972) time filter.

Moisture components are governed by the Kessler (1969) warm rain microphysics as implemented by Durran and Klemp (1983), and subgrid turbulence is parameterized using a 1.5-order closure scheme following the model of Sullivan et al. (1994). The model contains a run-time option to remove the conversion of cloud water to rain and the evaporation of rain from the original Kessler formulation. Both the condensation and evaporation of cloud water remain in the model.

\section{a. Initiating bubble and constant thermal methods}

The IB method for convection initiation (CI) involves the insertion of a positively buoyant perturbation into the horizontally homogeneous base-state environment (Klemp and Wilhelmson 1978; Carpenter et al. 1998). The buoyancy perturbation used in this study has a cosine-squared distribution within the IB volume and a peak magnitude of $1 \mathrm{~K}$. The perturbation is defined by

$$
\theta^{\prime}=\theta_{\max }^{\prime} \cos ^{2}\left[\frac{\pi}{2} \sqrt{\left(\frac{x-x_{c}}{x_{\mathrm{rad}}}\right)^{2}+\left(\frac{y-y_{c}}{y_{\mathrm{rad}}}\right)^{2}+\left(\frac{z-z_{c}}{z_{\mathrm{rad}}}\right)^{2}}\right]
$$

where $x_{c}, y_{c}$, and $z_{c}$, define the centroid of the bubble; $x_{\text {rad }}, y_{\text {rad }}$, and $z_{\text {rad }}$ are the radii of the bubble in the $x, y$, and $z$ directions, respectively; and $\theta_{\max }^{\prime}$ is the maximum magnitude of the perturbation, in this case, perturbation potential temperature. The model $\theta^{\prime}$ field is initialized using Eq. (1) for both the IB and constant thermal (CT) methods, but unlike the IB method, in which the buoyant bubble is prescribed only at the beginning of the simulation, the CT approach replaces the model solution values of $\theta^{\prime}$ within the initial bubble volume each large time step throughout the simulation with the $\theta^{\prime}$ values given by Eq. (1). This serves to provide a constant source of buoyancy to initiate deep convection, analogous to that observed over a heat island on a sunny day. Note that the diurnal variation of solar heating occurs on a time scale that is much longer than the convective time scale, so our constant source approximation is valid over the lifetime of convection reported in this paper $(\sim 2 \mathrm{~h})$. The time required to initiate convection via the IB or CT method is considerably less than that required by the approach of Carpenter et al. (1998), which is an important issue to consider for the proposed running of hundreds of numerical simulations. Note that the CT approach described above can 
also be characterized as a constant heat flux method, but prespecification of the flux is not desirable, because it would require the specification of the vertical motion in the source region. We prefer to allow the vertical motion field to develop according to the model equation set. The heat flux can be diagnosed during the simulation from the potential temperature perturbation and resultant vertical velocity located above the CT region.

\section{b. Low-level convergence}

For this study we have selected a simple low-level convergence wind field (LLC) that is prescribed via the velocity perturbation fields through the use of the incompressible mass continuity equation [Eq. (2)], where $u_{m}^{\prime}$ and $v_{m}^{\prime}$ are the magnitudes of the zonal and meridional mesoscale perturbation velocities, respectively, $w^{\prime}$ is the magnitude of the forced (mesoscale) vertical velocity, and $\mathrm{D}$ is the horizontal divergence:

$$
\frac{\partial u_{m}^{\prime}}{\partial x}+\frac{\partial v_{m}^{\prime}}{\partial y}=-\frac{\partial w^{\prime}}{\partial z}=D .
$$

The forcing layer velocity perturbations are derived by specifying the velocity potential, denoted $\phi_{m}(x, y, z)$, and assuming a Gaussian shape in the horizontal and a linear profile with height. The velocity potential is

$$
\begin{aligned}
\phi_{m}(x, y, z)= & A f(z) \exp \left[-\left(\frac{x-x_{c}}{\lambda_{x}}\right)^{2}\right] \\
& \times \exp \left[-\left(\frac{y-y_{c}}{\lambda_{y}}\right)^{2}\right],
\end{aligned}
$$

where $x_{c}$ and $y_{c}$ are the horizontal coordinates of the center of the ascent region, and $\lambda_{x}$ and $\lambda_{y}$ are "shape control parameters" of the divergence field in the $x$ and $y$ directions, respectively. Here, $A$ is a constant that determines the maximum forcing magnitude, and $f(z)$ is a linear function defined as,

$$
f(z)=1-\frac{z_{\mathrm{sfc}}-z}{z_{\mathrm{sfc}}-z_{\mathrm{deep}}},
$$

where $z_{\mathrm{sfc}}$ is the physical surface height and $z_{\text {deep }}$ is the depth of the forcing region. The shape control parameters are used to prescribe the horizontal width and ellipticity of the forcing region. From (4), the magnitude of $\phi_{m}$ is greatest at the surface, decreasing linearly to zero at the top of the forcing region. Finally, the components of the horizontal perturbation flow are given in terms of the velocity potential by

$$
\frac{\partial \phi_{m}}{\partial x}=u_{m}^{\prime}=-\frac{2 A\left(x-x_{c}\right)}{\lambda_{x}^{2}} e^{-\left(x-x_{c} / \lambda_{x}\right)^{2}} e^{-\left(y-y_{c} / \lambda_{y}\right)^{2}} f(z),
$$

and

$$
\frac{\partial \phi_{m}}{\partial y}=v_{m}^{\prime}=-\frac{2 A\left(y-y_{c}\right)}{\lambda_{y}^{2}} e^{-\left(x-x_{c} / \lambda_{x}\right)^{2}} e^{-\left(y-y_{c} / \lambda_{y}\right)^{2}} f(z) .
$$

Horizontal divergence is simply the horizontal Laplacian of $\phi_{m}$, and the maximum magnitude for the divergence $D_{\max }$ occurs at $\left(x_{c}, y_{c}, z_{\mathrm{sfc}}\right)$; thus, the constant $A$ is related to the divergence maximum according to

$$
A=\frac{-D_{\max }}{2}\left(\frac{1}{\lambda_{x}^{2}}+\frac{1}{\lambda_{y}^{2}}\right)^{-1} .
$$

The vertical velocity, pressure, temperature, and moisture fields are not directly constrained by this method. The perturbation $u$ and $v$ are specified according to (5) and (6) within the region of convergence. There is no specification of a matching divergence field as described by previous methods (Chang and Orville 1973; Chen and Orville 1980; Xin and Reuter 1996). Therefore, the wind fields at points outside of the convergence region are predicted according to the model's governing equations. This allows for a modeldeveloped divergence field aloft that is a combination of the response of the atmosphere to the forced lowlevel convergence and the anvil outflow associated with the convection. The LLC approach represents a source of momentum, and thus this method could be identified as a momentum flux method, as proposed by Crook and Moncrieff (1988, hereafter CM88). The difference between the CM88 study and our method is that we cannot prespecify the momentum flux magnitude because we do not have a fully compressible analytical solution for the vertical velocity. As is the case for the CT method, we can diagnose the vertical flux of horizontal momentum from the predicted solution.

Inclusion of the mesoscale perturbation velocities $u_{m}^{\prime}$ and $v_{m}^{\prime}$ at $t=0$ imposes an unbalanced state in the model and a response by the pressure, potential temperature, and vertical velocity as the time integration progresses. The total horizontal ( $u$ and $v$ ) components are a combination of a nondivergent base-state horizontal wind component ( $\bar{u}$ and $\bar{v})$ and the computed values of $u_{m}^{\prime}$ and $\bar{v}_{m}$. Both $u_{m}^{\prime}$ and $v_{m}^{\prime}$ were held constant throughout the simulation within the forcing region and the $\lambda_{x}$ and $\lambda_{y}$ constants were chosen to provide nearzero resultant $u_{m}^{\prime}$ and $v_{m}^{\prime}$ near the boundaries.

\section{Experiment setup and results}

Simulations were conducted to demonstrate differences between the IB, CT, and LLC initiating mechanisms on the development of deep, moist convection. 
TABLE 1. Select model constants and parameters for each type of forcing mechanism. Dtbig and Dtsml are the large and small model time-step lengths; $\alpha$ is the Asselin time smoothing constant; $C_{x}, C_{y}, C_{z}$ are computational mixing coefficients; $z_{r}$ is the height above the ground of the bottom of the Rayleigh damping region; and $R_{d}$ is the Rayleigh damping coefficient.

\begin{tabular}{cccccccc}
\hline \hline Forcing type & Dtbig $(\mathrm{s})$ & Dtsml $(\mathrm{s})$ & $\alpha\left(\mathrm{s}^{-1}\right)$ & $C_{x}, C_{y}\left(\mathrm{~s}^{-1}\right)$ & $C_{z}\left(\mathrm{~s}^{-1}\right)$ & $z_{r}(\mathrm{~m})$ & $R_{d}\left(\mathrm{~s}^{-1}\right)$ \\
\hline IB & $5.0 / 2.5$ & $0.5 / 0.25$ & 0.05 & 0.0005 & 0.0005 & 12000 \\
CT & 2.5 & 0.25 & 0.05 & 0.0005 & 0.0005 & 12000 \\
LLC & 5.0 & 0.5 & 0.05 & 0.0005 & 0.0005 & 12000 \\
\hline
\end{tabular}

The model domain size used in this study was $48 \mathrm{~km} \times$ $48 \mathrm{~km} \times 20 \mathrm{~km}$ in the $x, y$, and $z$ directions, respectively. The grid spacing was $400 \mathrm{~m} \times 200 \mathrm{~m}$ in the horizontal and vertical directions, respectively. Our justification for the $400 \mathrm{~m} \times 200 \mathrm{~m}$ grid spacing was based on a resolution that is capable of reproducing the general characteristics of moist, deep convection, such as the time of the onset of convection, overall cloud shape, and development and general cell regeneration, all of which are well represented in the $400 \mathrm{~m} \times 200 \mathrm{~m}$ grid spacing simulations. The computational mixing coefficients used for the simulations damp the smallest wavelength magnitudes every large time step to approximately $80 \%$ of their original value. A summary of the model parameters for each forcing type is presented in Table 1 . The model equations were integrated from $t=$ $0-150$ min to allow the deep, moist convection to evolve through several convective cycles, defined as distinct cloud regions with bubblelike appearance moving through a less intense (in terms of vertical velocity and buoyancy) region of sustained updraft. Longer time periods could be studied when more realistic features are modeled, including precipitation, cold pools, and sustained forcing that does not replace any portion of the time-dependent solution in the forcing region.

The background environment, a derivative from the 20 May 1977 Fort Sill, Oklahoma, sounding (Fig. 1), was modified to exhibit a weakly stable layer at the top of a neutrally stratified boundary layer and weak unidirectional shear from 850 to $300 \mathrm{hPa}$. The weak stable layer suppresses the weaker convection that developed in numerical simulations and allows only for stronger cells to develop. The PBL associated with the background environment was approximately $800 \mathrm{~m}$ deep and was not well mixed with respect to moisture. The level of free convection for a surface parcel was at a height of $710 \mathrm{~m}$, just below the stable layer, resulting in a maximum calculated value of convective available potential energy (CAPE) of approximately $2900 \mathrm{~J} \mathrm{~kg}^{-1}$ and a convective inhibition (CIN) of $0 \mathrm{~J} \mathrm{~kg}^{-1}$. The mixed layer, defined as the dry-adiabatic region just above the surface, has a CIN of approximately $80 \mathrm{~J}$ $\mathrm{kg}^{-1}$. The Brunt-Väisälä oscillation period for the layer between the LFC and the equilibrium level (EL) of the surface parcel is approximately 2 min. The arbitrary selection of the 20 May 1977 supercell sounding in this research does not limit us to the type of storms we wish to study. We selected this sounding because of its broad familiarity within the community, and we modified the wind field to fit the observed range of wind speed and shear for multicell thunderstorms. The thermodynamic variability in the sounding used in this study is less important in terms of storm-type classification and was varied to meet the needs of the parameter range study.

Model solutions for each experiment were examined via the distribution of cloud water mixing ratios $\left(q_{c}\right)$ and time series depictions of the domain-wide maximum vertical velocities $\left(w_{\max }\right)$, which were associated with the most intense convective updrafts within the main updraft region. Visual inspection of cloud mixing ratio and vertical velocity fields was found to be very useful in identifying discrete cells within the updraft, and both matched well with other indicators of discrete cell development and movement, such as vorticity generation, buoyancy, and resultant deformation (not shown). Tests were performed using the LLC forcing to determine the length of time in which the solution is unaffected by the lateral boundaries. Compared with runs using 4- and 16-times-larger horizontal domains, the solutions with the $48 \mathrm{~km} \times 48 \mathrm{~km}$ horizontal domain were almost identical for the first 75 min from the start of the simulation (30 min from the onset of convection). For the next $30 \mathrm{~min}$, the simulations exhibit very similar solutions in terms of phase and magnitude of maximum vertical velocity and liquid water content for the individual convective cells. After $105 \min (60$ min after the onset of deep convection), the maximum domain-wide vertical velocity contains noticeable differences between the tests, and we will not discuss the $48 \mathrm{~km} \times 48 \mathrm{~km}$ domain solutions after that time.

\section{a. IB and CT forcing experiment results}

In these experiments, the vertical radius $\left(z_{\mathrm{rad}}\right)$ and forcing center were varied (Table 2), and the potential temperature perturbation was specified to be either ellipsoidal or half ellipsoidal in shape. The value of $\theta_{\max }^{\prime}$ was located at $z_{c}=0 \mathrm{~m}$ (the surface) for the halfellipsoid forcing (HLF), and at a height of $z_{c}=z_{\mathrm{rad}}$ for 


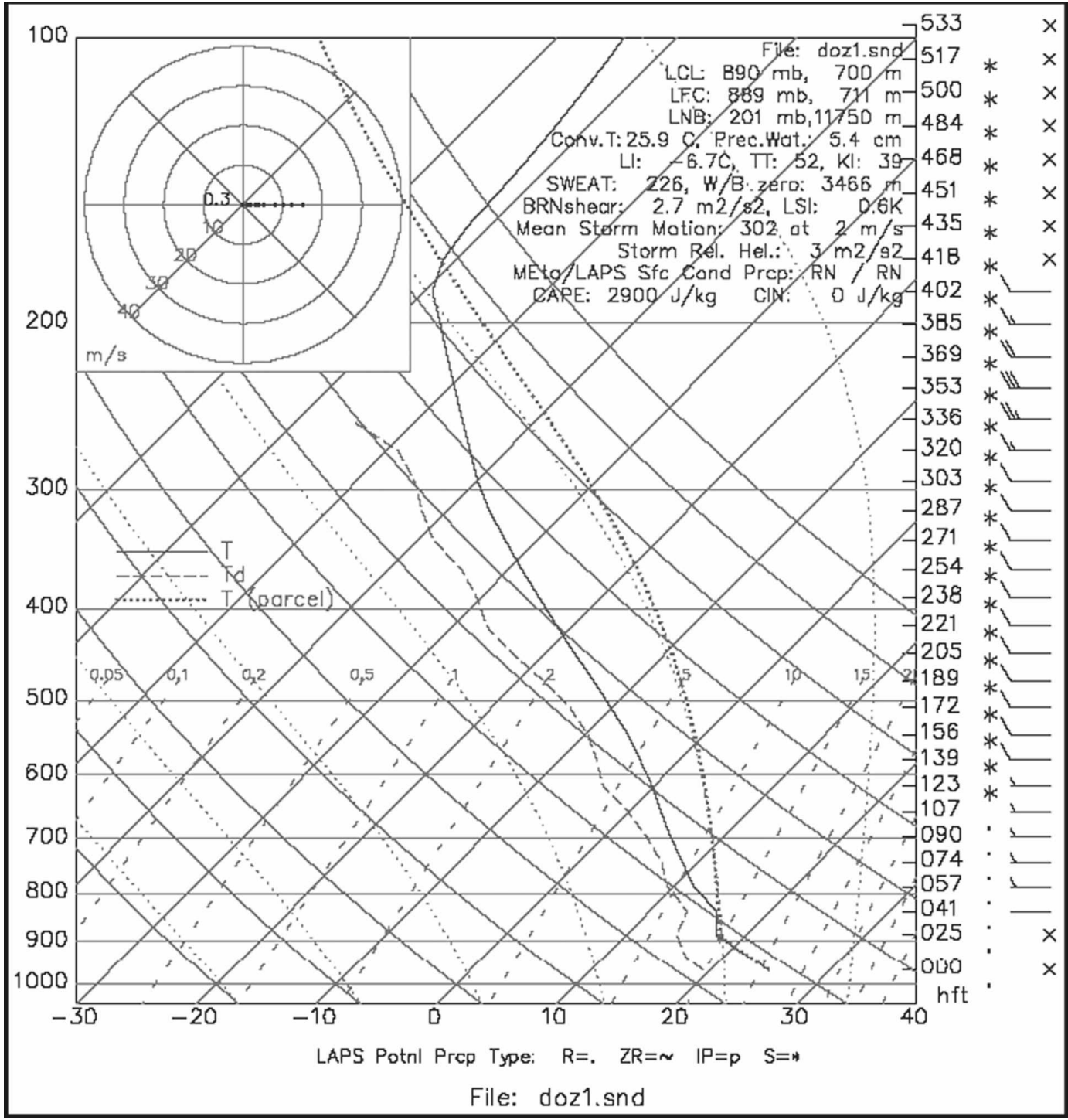

FIG. 1. Skew $T-\log p$ plot showing the background environment vertical profiles of temperature, moisture, and winds used in the simulations.

a full-ellipsoid perturbation (FUL), where $z_{c}$ denotes the height of the perturbation center. The IB cases were conducted using big time steps of $5 \mathrm{~s}$, with the exception of IBHLF5, for which the large time step was reduced to $2.5 \mathrm{~s}$ to accommodate for a stronger vertical velocity response. All CT cases employed a large time step of $2.5 \mathrm{~s}$.

The effects of using both half- and full-ellipsoidal perturbation regions to initiate deep, moist convection were examined using time series plots of $w_{\max }$ (Fig. 2). Greater peak values of $w_{\max }$ were observed with the convection initiated via IB or CT methods using the full-ellipsoid region than that initiated using halfellipsoid shapes (Fig. 2). This result is similar to that observed by Brooks (1992) in which bubbles located at slightly greater heights developed into more intense convection than bubbles located at lower heights; although he also varied the magnitude of the $\theta_{\max }^{\prime}$ of the IBs in conjunction with the vertical location of the bubble.

For the CT case, the $w_{\max }$ values for the full-ellipsoid regions (Fig. 2c) show a deep, moist convective response in all five cases. Initial peak updraft speeds of approximately $25 \mathrm{~m} \mathrm{~s}^{-1}$ were observed for source regions defined with vertical radii of $600-1000 \mathrm{~m}$. Slightly greater initial peak updraft speeds of approximately 35 and $40 \mathrm{~m} \mathrm{~s}^{-1}$ were evident as $z_{\text {rad }}$ of the source region increases to 1200 and $1400 \mathrm{~m}$, respectively. This is con- 
TABLE 2. IB and CT experiment designations and associated forcing parameter settings: $x_{\text {rad }}, y_{\text {rad }}$, and $z_{\text {rad }}$ are the $x$ direction, $y$ direction, and vertical radii of the source region and $z_{c}$ is the distance above the ground surface of the forcing region.

\begin{tabular}{cccccccc}
\hline \hline Full ellipsoid & $z_{\text {rad }}(\mathrm{m})$ & $z_{c}(\mathrm{~m})$ & $x_{\text {rad }}, y_{\text {rad }}(\mathrm{m})$ & Half ellipsoid & $z_{\text {rad }}(\mathrm{m})$ & $z_{c}(\mathrm{~m})$ & $x_{\text {rad }}, y_{\text {rad }}(\mathrm{m})$ \\
\hline IBFUL1 & 300 & 300 & 5000 & IBHLF1 & 600 & 0 & 5000 \\
IBFUL2 & 400 & 400 & 5000 & IBHLF2 & 800 & 0 & 5000 \\
IBFUL3 & 500 & 500 & 5000 & IBHLF3 & 1000 & 0 & 5000 \\
IBFUL4 & 600 & 600 & 5000 & IBHLF4 & 1200 & 0 & 5000 \\
IBFUL5 & 700 & 700 & 5000 & IBHLF5 & 1400 & 0 & 5000 \\
CTFUL1 & 300 & 300 & 5000 & CTHLF1 & 600 & 0 & 5000 \\
CTFUL2 & 400 & 400 & 5000 & CTHLF2 & 800 & 0 & 5000 \\
CTFUL3 & 500 & 500 & 5000 & CTHLF3 & 1000 & 0 & 5000 \\
CTFUL4 & 600 & 600 & 5000 & CTHLF4 & 1200 & 0 & 5000 \\
CTFUL5 & 700 & 700 & 5000 & CTHLF5 & 1400 & 0 & 5000 \\
\hline
\end{tabular}

sistent with that described by Weisman et al. (1997) in which analytical solutions to a linearized set of equations were used to discuss the differences between hydrostatic and nonhydrostatic motions; deeper disturbances produce stronger vertical motion responses. Figures $2 \mathrm{a}, \mathrm{b}$ also show that the IB convection takes longer to develop when initiated with a whole versus a half ellipsoid. For the IB whole-bubble cases (Fig. 2a), in- creasing the vertical radius of the perturbed region appears to increase the amount of time required for initiation of deep, moist convection. The vertical extent of the IB does not appear to have much of an effect on the resulting values of $w_{\max }$, because similar peak magnitudes were attained in nearly all of the IB cases (Figs. 2a,b), contrary to the results of the CT tests (Figs. 2c,d). Results from McPherson and Droegemeier

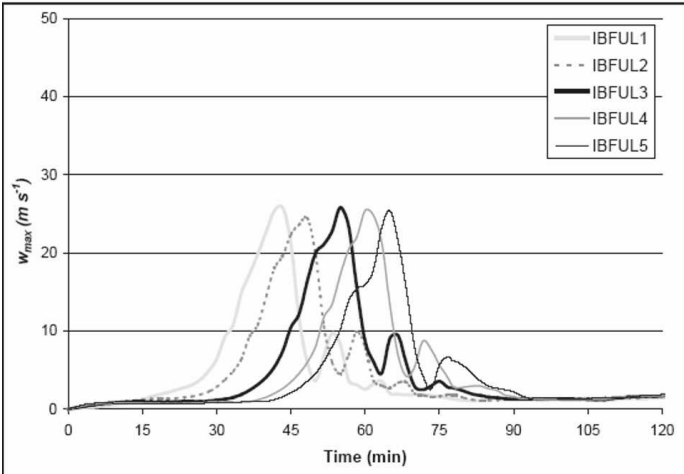

(c)

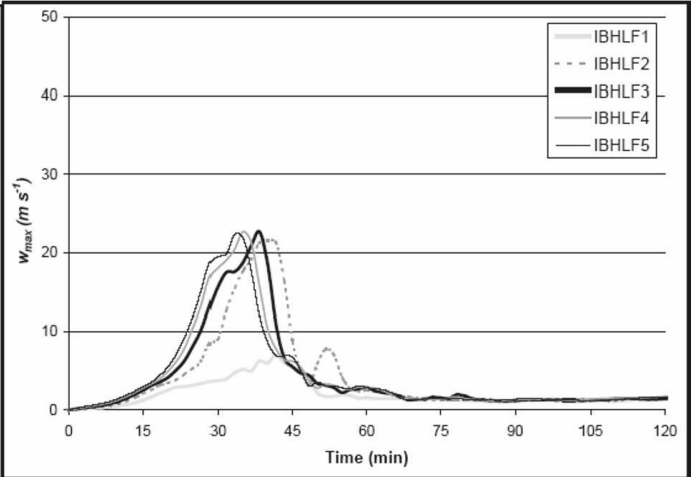

(d)
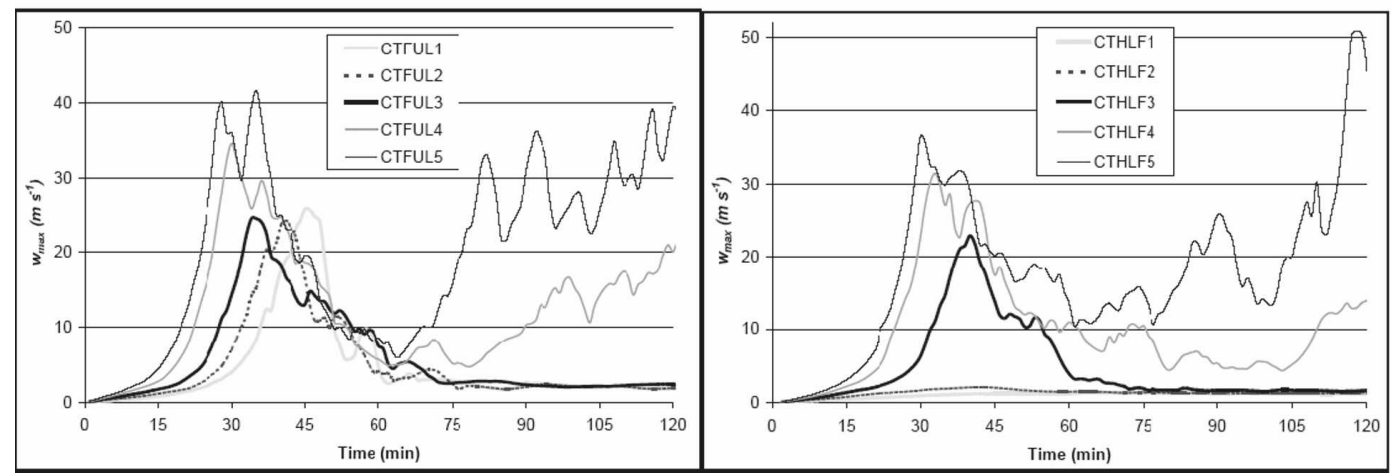

FIG. 2. Time series of domain-wide $w_{\max }\left(\mathrm{m} \mathrm{s}^{-1}\right)$ values for (a) full- and (b) half-ellipsoid perturbation IB experiments and (c) full- and (d) half-ellipsoid-shaped CT experiments. 
(1991) and Brooks (1992) suggest that, for equal magnitudes of $\theta_{\max }^{\prime}$, initial convective intensity may be much more sensitive to the width rather than the depth of the IB. According to Weisman et al. (1997), both narrower and taller source regions will contribute to stronger responses. In all IB experiments, deep, moist convection is not sustained beyond the initial convective development as expected, owing to the following two possible mechanisms: 1) the absence of precipitation and the development of a cold pool in the simulations, and 2) the presence of a stable layer near the surface. Additional tests were conducted that included precipitation physics. Sustained convection was observed when the IB horizontal radii were increased by a factor of 2 , the vertical radius and center location was increased to $1.5 \mathrm{~km}$, and the magnitude increased by a factor of 4 (not shown).

As shown in Figs. 2c,d, sustained deep, moist convection resulting from $\mathrm{CT}$ forcing does not develop for forcing regions with the smallest vertical diametersCTHLF1 and CTHLF2-in which peak $w_{\max }$ values of only about $3 \mathrm{~m} \mathrm{~s}^{-1}$ were observed. As the vertical extent of the perturbation increases, the time required for the initial deep, moist convection to develop decreases, consistent with current theory (Weisman et al. 1997). Unlike the results for the IB cases, this result seems to be independent of the vertical location of $\theta_{\text {max }}^{\prime}$. The regeneration period, defined as the elapsed time between two subsequent peaks in $w_{\max }$, was estimated for both the full- and half-ellipsoidal $\mathrm{CT}$ cases to be roughly $8-10 \mathrm{~min}$ for the cases with the higher placement of the center compared to the lower-centerplaced tests. For the half-ellipsoid CT cases, the $w_{\max }$ response was weaker, with longer regeneration periods than in the full-ellipsoid scenarios (by approximately 15 min). During the CT experiments, the strong updrafts created a return flow that pulled drier air down, as is evident from the vertical cross section of the water vapor mixing ratio air (not shown), into the periphery of the source region. These air motions increased the entrainment of drier air into the moist source region, reducing the potential instability of the source region, and prevented strong, sustained convection in the weaker and smaller source region cases. A trajectory analysis is planned for a future manuscript that shows the interactions of the source region with the environment more clearly.

\section{b. Low-level convergence experiment results}

The LLC forcing parameters are presented in Table 3 and were chosen to provide insight regarding model convective responses as a function of the geometry and strength in this limited study. All LLC cases employed
TABLE 3. LLC experiment designation and associated forcing parameter settings; $D_{\max }$ is the maximum divergence, $z_{\text {deep }}$ is the depth of the forcing region, and $\lambda_{x}, \lambda_{y}$ are constants used to control the magnitude of the forced horizontal velocities near the boundaries.

\begin{tabular}{lccc}
\hline \multicolumn{1}{c}{ Run name } & $D_{\max }\left(\mathrm{s}^{-1}\right)$ & $z_{\text {deep }}(\mathrm{m})$ & $\lambda_{x}, \lambda_{y}(\mathrm{~m})$ \\
\hline CONTROL & $-1.0 \times 10^{-3}$ & 1000 & 5000 \\
CONVMAG1 & $-1.0 \times 10^{-4}$ & 1000 & 5000 \\
CONVMAG2 & $-5.0 \times 10^{-4}$ & 1000 & 5000 \\
CONVMAG3 & $-7.5 \times 10^{-4}$ & 1000 & 5000 \\
CONVMAG4 & $-2.5 \times 10^{-3}$ & 1000 & 5000 \\
CONVMAG5 & $-5.0 \times 10^{-3}$ & 1000 & 5000 \\
CONVDEEP1 & $-1.0 \times 10^{-3}$ & 600 & 5000 \\
CONVDEEP2 & $-1.0 \times 10^{-3}$ & 800 & 5000 \\
CONVDEEP3 & $-1.0 \times 10^{-3}$ & 1200 & 5000 \\
CONVDEEP4 & $-1.0 \times 10^{-3}$ & 1400 & 5000 \\
CONVWIDE1 & $-1.0 \times 10^{-3}$ & 1000 & 2500 \\
CONVWIDE2 & $-1.0 \times 10^{-3}$ & 1000 & 7500 \\
CONVWIDE3 & $-1.0 \times 10^{-3}$ & 1000 & 10000 \\
\hline
\end{tabular}

a large time step of $5 \mathrm{~s}$. A future paper will report on 144 simulations that cover a more extensive forcing and environmental conditions parameter space.

\section{1) Sensitivity to divergence magnitude}

Tests using the low-level convergence method and varying only the divergence magnitude are designated CONVMAG. As the forcing magnitude $\left(D_{\max }\right)$ increases (Fig. 3, top panel), the time required for deep, moist convection to become established decreases and the maximum updraft velocity increases. This result agrees qualitatively with Xin and Reuter (1996), who found that an earlier onset of surface rainfall (and, therefore, an earlier onset of deep, moist convection) was associated with an increase in the magnitude of low-level convergence. Thus, the intensity of the deep, moist convection is related to the maximum divergence magnitude $\left(D_{\max }\right)$ for simulations in which the volume of the forcing region remains constant. This sensitivity is also consistent with results found by Tripoli and Cotton (1980). For the tests conducted in the current study, once deep, moist convection develops, recurrent convective cells develop in all of the simulations presented in Fig. 3, except for CONVMAG1. As the forcing magnitude increases from $\left|-5.0 \times 10^{-4} \mathrm{~s}^{-1}\right|$ to $\mid-5.0 \times$ $10^{-3} \mathrm{~s}^{-1} \mid$, the regeneration period decreases from approximately 20 to $10 \mathrm{~min}$. Animations of the cloud water mixing ratios for these cases visually confirm this decrease in cell regeneration period with increasing values of $D_{\max }$ (not shown).

\section{2) SEnsitivity to FORCING LAYER DEPTH}

Low-level convergence tests varying only the forcing layer depth are designated CONVDEEP. Convective initiation is observed to occur at similar times (Fig. 3, 

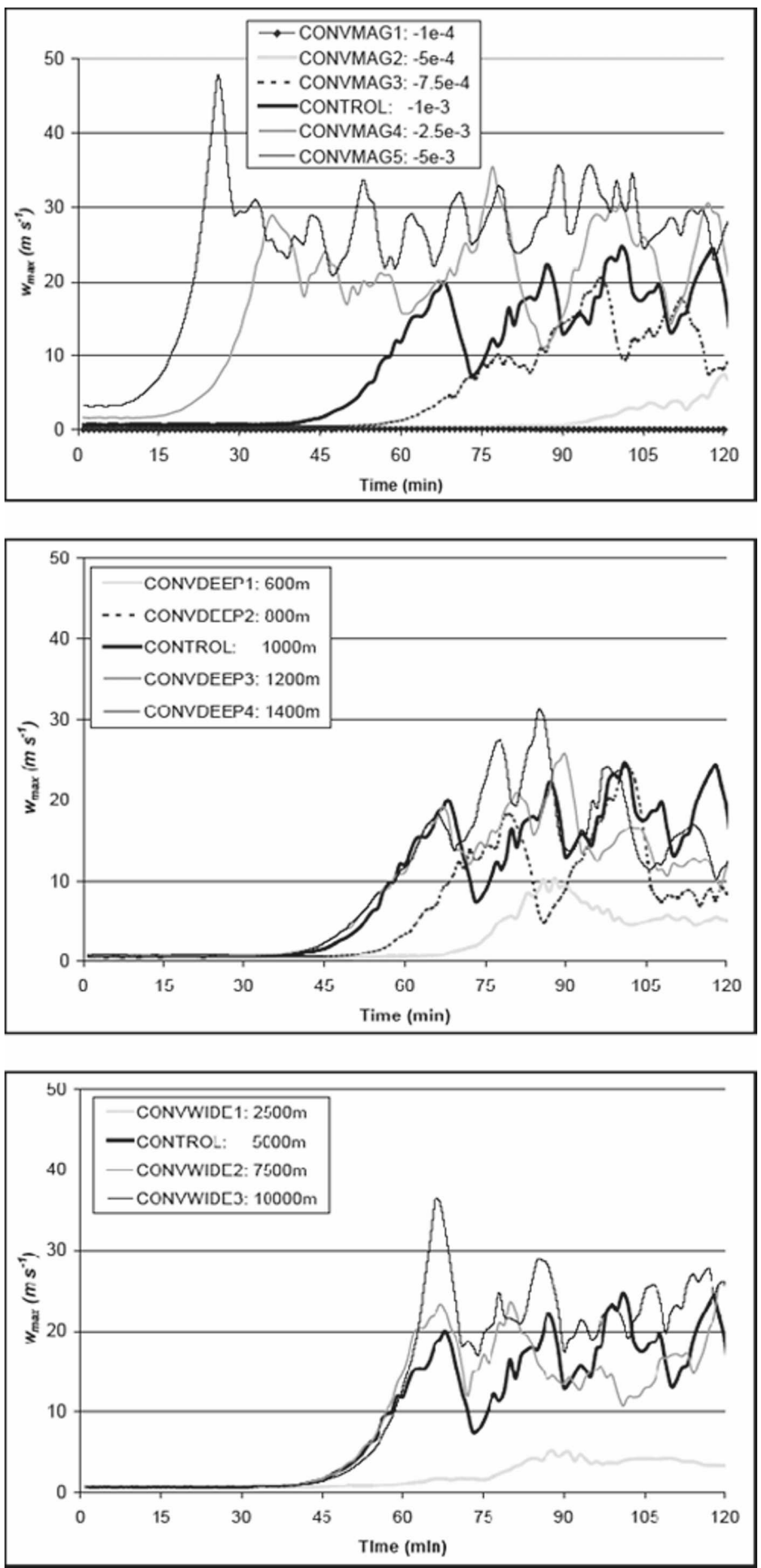

FIG. 3. Time series of $w_{\max }$ for LLC forcing simulations in which the (top) forcing magnitude divergence, (middle) forcing layer depth, and (bottom) horizontal width $\lambda_{x}=\lambda_{y}$ of the forcing region are varied. 
middle panel) for the CONVDEEP3 and CONVDEEP4 cases, in which the forcing layer depths range from 1200 to $1400 \mathrm{~m}$ with a constant divergence magnitude $\mathrm{I}-1.0$ $\times 10^{-3} \mathrm{~s}^{-1} \mid$. As the forcing layer depth is decreased below $1000 \mathrm{~m}$ (CONVDEEP2 and CONVDEEP1), however, convective initiation is delayed until later in the simulation, as found by Xin and Reuter (1996). However, the numerical model and the forcing characteristics applied in the current work were quite different from those of the aforementioned study. In the current study, the deeper forcing layers extend above the PBL top, such that air parcels within the stable layer are incorporated into the forced ascent region by the convergent flow acting to decrease the local stability in the PBL. The regeneration period decreases slightly, during the 65- and 105-min intervals, as the forcing layer depth increases (Fig. 3, middle panel). Note that these results are consistent with scale analysis, given that the time scale is proportional to the horizontal length scale divided by the vertical velocity scale. As the forcing layer depth increases, the resultant integrated vertical velocity increases, and therefore the onset of convection should decrease, consistent with current buoyancyforced vertical motion theory (Weisman et al. 1997).

\section{3) Sensitivity to horizontal FORCING REgion SIZE}

Low-level convergence tests performed by varying the horizontal forcing region size are designated CONVWIDE. Deep, moist convection is observed for all but the smallest horizontal radii forcing case (Fig. 3, bottom panel; CONVWIDE1), in which peak magnitudes of $w_{\max }$ attain values of only $5 \mathrm{~m} \mathrm{~s}^{-1}$ by $90 \mathrm{~min}$. As the width of the horizontal radii increases, convective intensity increases and the cell regeneration period decreases. These results suggest that convection is sensitive to the horizontal width of the convergence region for a specified forcing magnitude and layer depth. This is consistent with results from CM88, who demonstrated that the air within the forcing region experienced a larger total vertical displacement for wider convergence zones than for narrower forcing regions, causing parcels to undergo longer periods of forced ascent, and eventually leading to more vigorous convective updrafts.

The time required for initiation to occur does not appear to be affected by the width of the convergence region. The divergence magnitude of the forced ascent is largely independent of $\lambda_{x}$ and $\lambda_{y}$, because although the horizontal area over which air is forced to rise varies, the maximum divergence magnitude of this forced ascent was equal in all cases. Convective updrafts continue to redevelop throughout the simulations (Fig. 3, bottom panel), with wider forcing regions resulting in a decrease in the average regeneration period of the convective updrafts.

\section{c. Convection initiation method intercomparison}

We now compare three different initiating mechanism experiments, each of which use similar initial spatial perturbation dimensions of $10 \mathrm{~km} \times 10 \mathrm{~km} \times 1.2$ $\mathrm{km}$ in the horizontal and vertical directions. The names of the cases are IBHLF4, CTHLF4 (Table 2), and CONVDEEP3 (Table 3 ). The maximum potential temperature perturbation $\left(\theta_{\max }^{\prime}\right)$ in the IB and CT cases and the maximum divergence magnitude in the LLC case were located at the surface. The perturbations vanish at a height of $1.2 \mathrm{~km}$ in each case. We found that the plan view conveys less information than vertical cross sections, because one can see the development of a cell and the correlation between vertical velocity, temperature, and moisture perturbations better with the cross section than with the plan view. Vertical cross sections through the center of the domain in the $y$ direction of the $q_{c}$ and vertical velocity fields (Figs. 4,5) depict the early and later stages of convective development for the three cases and show the consistency between the discrete cloud elements and the associated vertical motion. Results are presented at different times in the LLC case because the initial convection produced via a convergent flow field takes longer to develop. Differences exist in the structure of the convective clouds produced by the different initiation mechanisms, especially in the later phases of development (Fig. 5). The elapsed simulation time between the early and later phases of convection for each case was $21 \mathrm{~min}$. However, the evolution of the deep, moist convection in each simulation was remarkably dissimilar within this time frame (not shown).

In the IB case (Figs. 5a,d), the deep, moist convection is in its dissipating stage and only the upper portion of the original cloud remains. The CT simulation (Figs. $5 b, e)$ shows the deep, moist convection in a more mature stage, consisting of several updrafts in close proximity to one another, such that they appear to be merged into a single updraft or plume. The distribution of $q_{c}$ and $w$ in the LLC simulation (Figs. 5c,f) is associated with three separate convective entities, suggesting that the deep, moist convection occurred in several discrete stages or bubbles. In general, deep, moist convection generated in the CTHLF4 case attains the greatest peak magnitude of $w_{\max }$ among the three simulations at $29 \mathrm{~m} \mathrm{~s}^{-1}$ (Fig. 6), and the peak $w_{\max }$ value is slightly larger in CONVDEEP3 than in IBHLF4 (26 versus $23 \mathrm{~m} \mathrm{~s}^{-1}$ ). Therefore, the intensity and character of the deep, moist convection is evidently quite sensi- 
(a)

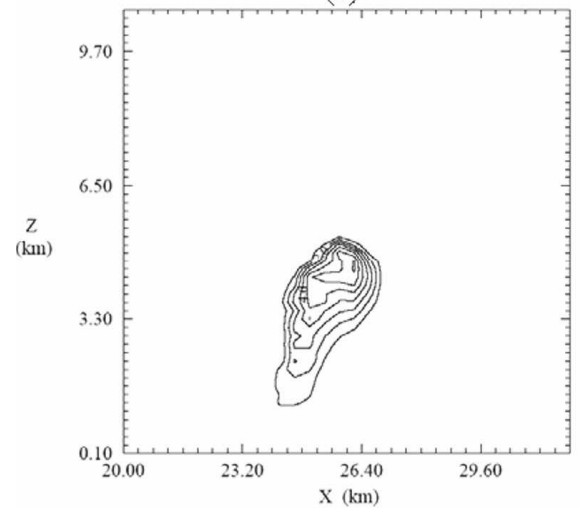

(b)

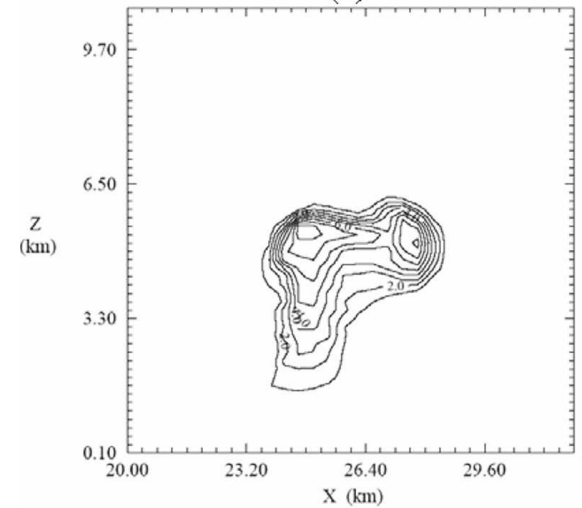

(c)

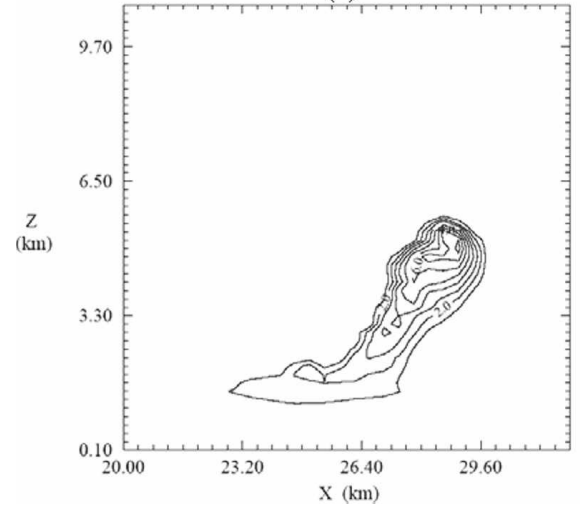

(d)

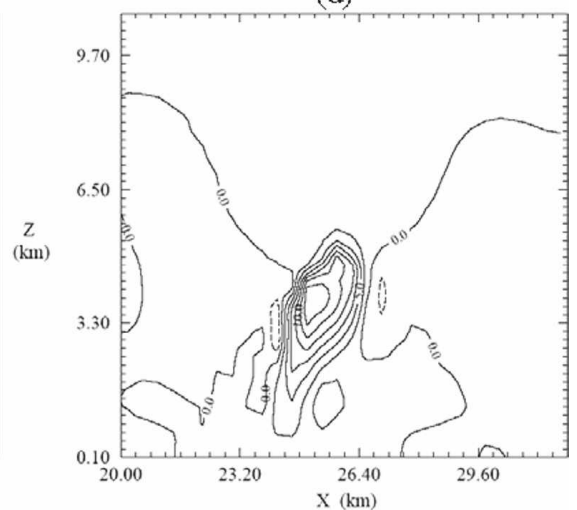

(e)

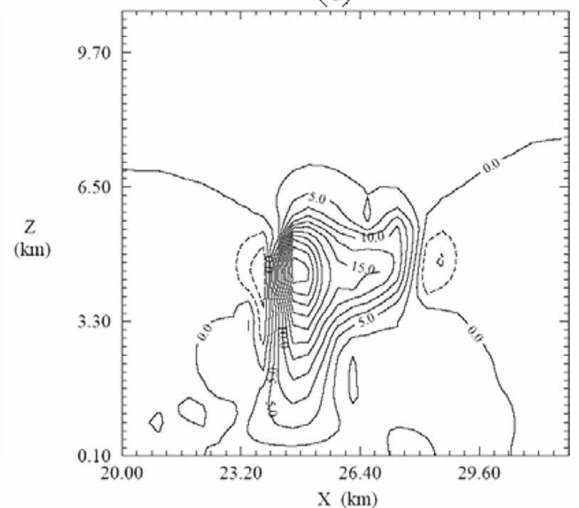

(f)

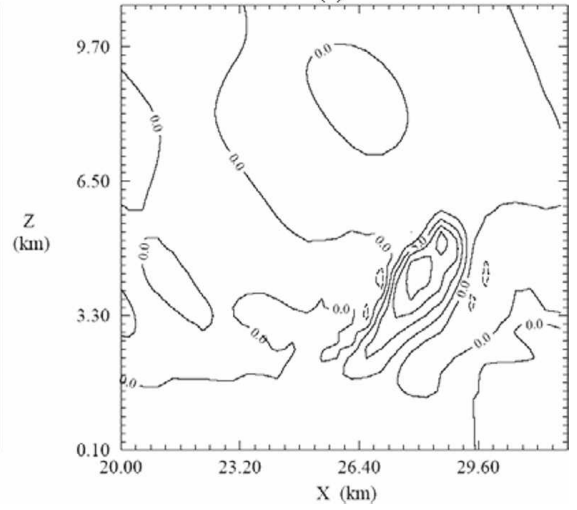

FIG. 4. The $x-z$ cross sections of (left) cloud water content (contour interval $1 \mathrm{~g} \mathrm{~kg}^{-1}$ ) and (right) vertical velocity (contour interval $2.5 \mathrm{~m} \mathrm{~s}^{-1}$ ) for an (a), (d) IB, (b), (e) CT, and (c), (f) LLC case at simulation times $t=30 \mathrm{~min}$ for the top two rows and $t=60 \mathrm{~min}$ for the bottom row. Cross sections taken through center of initial forcing region $(y=24 \mathrm{~km})$.

tive to the CI mechanism. The deep, moist convection in IBHLF4 only exhibits one convective cycle between approximately $15-$ and $50-\mathrm{min}$ simulation times. The CTHLF4 case only produced one significant convective cycle, as measured by $w_{\max }$, during this time as well. However, the period of the cycle in this CT simulation appears to last longer than in the IB case, as shown by the slower rate of decrease in the $w_{\max }$ magnitudes between 35 and 65 min (Fig. 6) and a continuous cloudy updraft evident from just above the forcing to the anvil (Figs. $5 \mathrm{~b}, \mathrm{e}$ ). Several separate peaks in $w_{\max }$ were evident in the CONVDEEP3 case between 60 and $90 \mathrm{~min}$, corresponding to the multiple discrete and separate updrafts (Figs. 5c,f).

\section{Summary and future work}

The present work extends the studies of CM88 to three dimensions and uses sustained nonlinear forcing. 
(a)

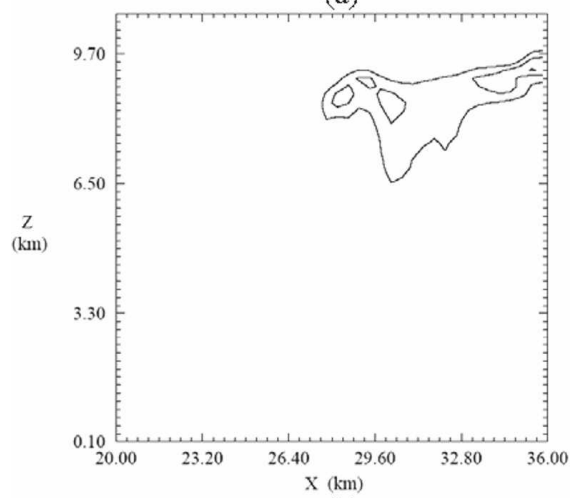

(b)

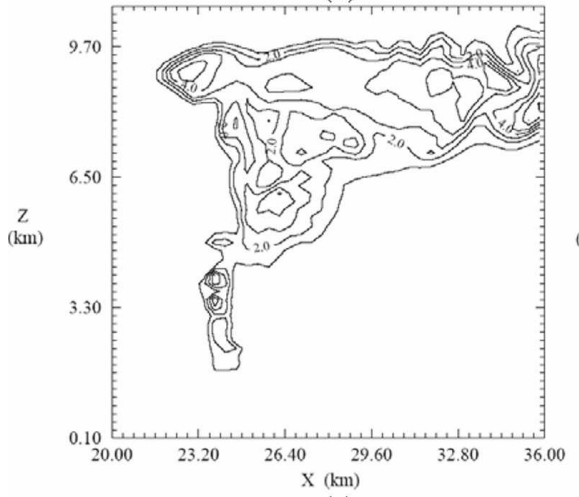

(c)

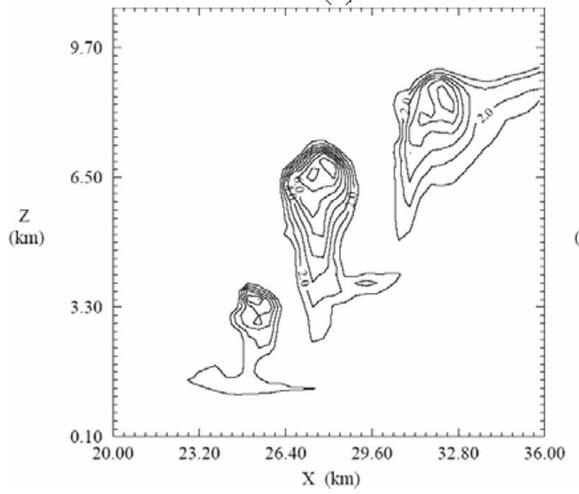

(d)

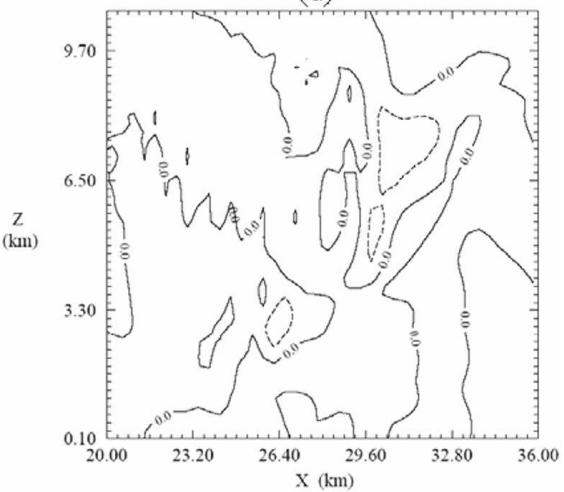

(e)

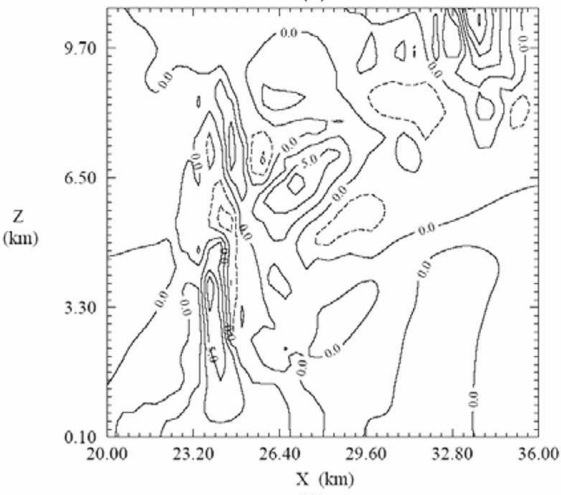

(f)

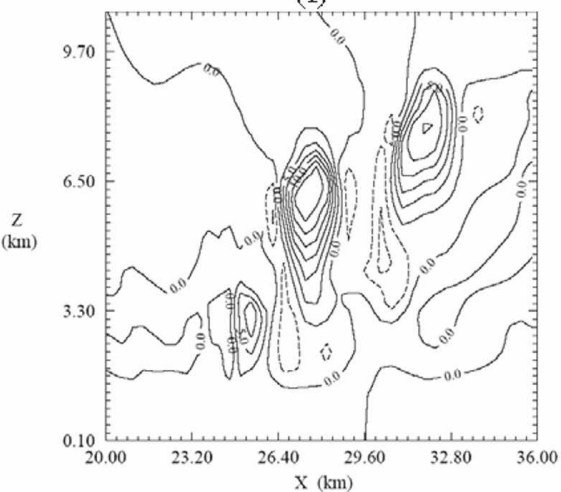

FIG. 5. As in Fig. 4, but at simulation times $t=51 \mathrm{~min}$ for top two rows, and $t=81 \mathrm{~min}$ for bottom row.

For the majority of the simulations presented here, both the CT and LLC continuous forcing methods were able to produce and sustain isolated nonprecipitating moist, deep convection in a weak CIN environment. All three initiating methods that were tested produced deep convective responses that displayed sensitivities to the initiation, intensity, and regeneration of convective elements as a function of the source strength, location, and geometry. Increases in the magnitude and depth of the LLC enhanced the convective response in terms of stronger vertical velocity, decreased time to initiate convection, and shorter cell regeneration periods, while increased width of the source region enhanced the vertical velocity magnitude. Increases in the depth of the CT forcing region enhanced the magnitude of the response and reduced the time to initiation and the period of cell regeneration, as diagnosed by maximum updraft velocity and liquid cloud water distributions. For the IB method, increases in the depth of the forcing region reduced the time to convection but did not impact the magnitude of the response as measured by the domain-wide vertical velocity magnitude. All IB simu- 


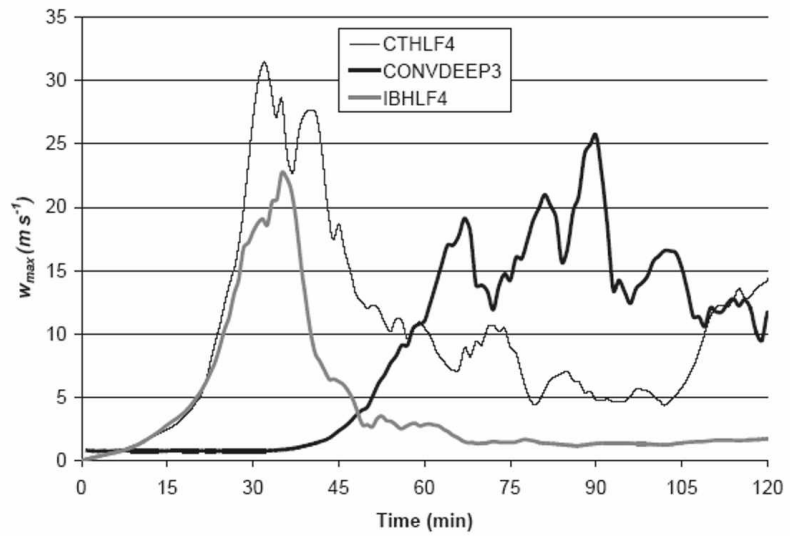

FIG. 6. Time series of $w_{\max }\left(\mathrm{m} \mathrm{s}^{-1}\right)$ for simulations using three different CI methods shown in Figs. 4 and 5. CT (dashed), LLC (solid), and IB (dotted) cases are shown.

lations demonstrated both a lack of redevelopment of convection without precipitation-induced cold pools and a significantly larger and stronger initial source region than that used herein.

Note that the work presented in this report is preliminary and that a second paper, summarizing results from an additional 192 simulations using the CT and LLC methods performed at a suggested $100-\mathrm{m}$ grid spacing (Bryan et al. 2003), will address the sensitivity of initiation and regeneration of isolated multicell convection as a function of CAPE, wind shear, and source region geometry and strength. In future work we will also investigate modifications to the CT and LLC methods that include a surface-based heat flux source and less restrictive LLC mechanisms that allow the perturbation horizontal velocity field to freely respond to the induced flow.

Acknowledgments. This research has been supported by the National Science Foundation under Grant ATM-0350539 and the University Of Oklahoma School of Meteorology. We also have received support from the Pittsburgh Supercomputing Center, the National Center for Supercomputing Applications, and the Oklahoma Supercomputing Center for Education and Research. We also thank Alan Shapiro, Evgeni Fedorovich, Lance Leslie, and three anonymous reviewers for several helpful suggestions regarding this work.

\section{REFERENCES}

Asselin, R., 1972: Frequency filter for time integrations. Mon. Wea. Rev., 100, 487-490.

Brooks, H. E., 1992: Operational implications of the sensitivity of modeled thunderstorms to thermal perturbations. Preprints, Fourth AES/CMOS Workshop on Operational Meteorology, Whistler, BC, Canada, Atmospheric and Environmental Service and Canadian Meteorological and Oceanographic Society, 398-407.

Bryan, G. H., J. C. Wyngaard, and J. M. Fritsch, 2003: Resolution requirements for the simulation of deep moist convection. Mon. Wea. Rev., 131, 2394-2416.

Carpenter, R. L., Jr., K. K. Droegemeier, and A. M. Blyth, 1998: Entrainment and detrainment in numerically simulated cumulus congestus clouds. Part I: General results. J. Atmos. Sci., 55, 3417-3432.

Chang, S. W., and H. D. Orville, 1973: Large-scale convergence in a numerical cloud model. J. Atmos. Sci., 30, 947-950.

Chen, C., and H. D. Orville, 1980: Effects of mesoscale convergence on cloud convection. J. Appl. Meteor., 19, 256-274.

Crook, N. A., and M. W. Moncrieff, 1988: The effect of large-scale convergence on the generation and maintenance of deep moist convection. J. Atmos. Sci., 45, 3606-3624.

Crum, T. D., R. B. Stull, and E. W. Eloranta, 1987: Coincident lidar and aircraft observations of entrainment into thermals and mixed layers. J. Climate Appl. Meteor., 26, 774-788.

Doswell, C. A., III, 1987: The distinction between large-scale and mesoscale contribution to severe convection: A case study example. Wea. Forecasting, 2, 3-16.

- , and P. M. Markowski, 2004: Is buoyancy a relative quantity? Mon. Wea. Rev., 132, 853-863.

Durran, D. R., and J. B. Klemp, 1983: A compressible model for the simulation of moist mountain waves. Mon. Wea. Rev., 111, 2341-2361.

Kessler, E., 1969: On the Distribution and Continuity of Water Substance in Atmospheric Circulations. Meteor. Monogr., No. 32, Amer. Meteor. Soc., 84 pp.

Klemp, J. B., and R. B. Wilhelmson, 1978: The simulation of three-dimensional convective storm dynamics. J. Atmos. Sci., 35, 1070-1096.

McNider, R. T., and F. J. Kopp, 1990: Specification of the scale and magnitude of thermals used to initiate convection in cloud models. J. Appl. Meteor., 29, 99-104.

McPherson, R. A., and K. K. Droegemeier, 1991: Numerical predictability experiments of the May 201977 Del City, OK supercell storm. Preprints, Ninth Conf. on Numerical Weather Prediction, Denver, CO, Amer. Meteor. Soc., 734-738.

Schlesinger, R. E., 1975: A three-dimensional numerical model of an isolated deep convective cloud: Preliminary results. J. Atmos. Sci., 32, 934-957.

Stensrud, D. J., G. S. Manikin, E. Rogers, and K. E. Mitchell, 1999: Importance of cold pools to NCEP mesoscale Eta Model forecasts. Wea. Forecasting, 14, 650-670.

Sullivan, P. P., J. C. McWilliams, and C. H. Moeng, 1994: A subgrid-scale model for large eddy simulations of planetary boundary layer flows. Bound.-Layer Meteor., 71, 247-276.

Tripoli, G. J., and W. R. Cotton, 1980: A numerical investigation of several factors contributing to the observed variable intensity of deep convection over South Florida. J. Appl. Meteor., 19, 1037-1063.

Weber, D. B., 1997: An investigation of the diurnal variability of the central Colorado downslope windstorm. Ph.D. dissertation, University of Oklahoma, $242 \mathrm{pp}$. [Available from School of Meteorology, University of Oklahoma, 120 David L. Boren Blvd., Suite 5900, Norman, OK 73072-7307.]

Weisman, M. L., W. C. Skamarock, and J. B. Klemp, 1997: The 
resolution dependence of explicitly modeled convective systems. Mon. Wea. Rev., 125, 527-548.

Wilhelmson, R. B., and L. J. Wicker, 2002: Numerical modeling of severe storms. Severe Convective Storms, Meteor. Monogr., No. 50, Amer. Meteor. Soc., 123-166.

Xin, L., and G. W. Reuter, 1996: Numerical simulation of the effects of mesoscale convergence on convective rain showers. Mon. Wea. Rev., 124, 2828-2842.
Xue, M., K. K. Droegemeier, and V. Wong, 2000: The Advanced Regional Prediction System (ARPS) - A multiscale nonhydrostatic atmospheric simulation and prediction tool. Part I: Model dynamics and verification. Meteor. Atmos. Phys., 75, 161-193.

Young, G. S., 1988: Turbulence structure of the convective boundary layer. Part II: Phoenix 78 aircraft observations of thermals and their environment. J. Atmos. Sci., 45, 727-735. 